\title{
Comparison of Gait Speed and Peripheral Nerve Function Between Chronic Kidney Disease Patients With and Without Diabetes
}

\author{
Seung Hwan Jin, $\mathrm{MD}^{1}$, Young Sook Park, $\mathrm{MD}^{1}$, Yun Hee Park, $\mathrm{MD}^{1}$, \\ Hyun Jung Chang, $\mathrm{MD}^{1}$, Sung Rok Kim, $\mathrm{MD}^{2}$

\begin{abstract}
${ }^{1}$ Department of Physical Medicine and Rehabilitation, Samsung Changwon Hospital, Sungkyunkwan University School of Medicine, Changwon; ${ }^{2}$ Division of Nephrology, Department of Internal Medicine, Samsung Changwon Hospital, Sungkyunkwan University School of Medicine, Changwon, Korea
\end{abstract}

\begin{abstract}
Objective To compare overall physical function, including gait speed and peripheral nerve function, between diabetic chronic kidney disease (CKD) patients and nondiabetic CKD patients and to investigate the association between gait speed and peripheral nerve function in CKD patients.

Methods Sixty adult CKD patients (35 with and 25 without diabetes), who received maintenance hemodialysis (HD), were included in this study. Demographic data, past medical history, current medical condition and functional data-usual gait speed, vibration perception threshold for the index finger (VPT-F) and the great toe (VPT-T), activity of daily living (ADL) difficulty, and peripheral neuropathy (PN) along with the degree of its severity-were collected and compared between the two groups. Correlations between the severity of PN and the impairment of other functions were identified.

Results Diabetic CKD patients showed significantly slower gait speed ( $\mathrm{p}=0.029)$, impaired sensory function (VPT-F, $\mathrm{p}=0.011$; VPT-T, $\mathrm{p}=0.023$ ), and more frequent and severe PN (number of PN, $\mathrm{p}<0.001$; severity of $\mathrm{PN}, \mathrm{p}<0.001$ ) as compared to those without diabetes. Usual gait speed had a significant negative correlation with the severity of PN (rho=-0.249, p=0.013). By contrast, VPT-F ( $\mathrm{rho}=0.286, \mathrm{p}=0.014)$ and VPT-T (rho=0.332, $\mathrm{p}=0.035$ ) were positively correlated with the severity of PN. ADL difficulty was comparatively more frequent in the patients with more severe $\mathrm{PN}(\mathrm{p}=0.031)$.

Conclusion In CKD patients with maintenance HD, their gait speed, sensory functions, and peripheral nerve functions were all significantly impaired when they have diabetes, and the severity of PN was negatively correlated with their gait speed, sensory function, and ADL function. Adverse effects of diabetes impacted physical performance of CKD patients. The physical disability of those patients might be attributable to PN and its severity.
\end{abstract}

Keywords Chronic kidney disease, Diabetes, Gait speed, Peripheral neuropathy

Received May 9, 2016; Accepted July 20, 2016

Corresponding author: Young Sook Park

Department of Physical Medicine and Rehabilitation, Samsung Changwon Hospital, Sungkyunkwan University School of Medicine, 158 Paryong-ro, Masanhoewon-gu, Changwon 51353, Korea. Tel: +82-55-290-6390, Fax: +82-55-290-6588, E-mail: jijibaeheiwon@hanmail.net

ORCID: Seung Hwan Jin (http://orcid.org/0000-0002-1585-1313); Young Sook Park (http://orcid.org/0000-0003-2752-7120); Yun Hee Park (http:// orcid.org/0000-0003-4707-2266); Hyun Jung Chang (http://orcid.org/0000-0001-9126-4316); Sung Rok Kim (http://orcid.org/0000-0002-8065-6629).

(c) This is an open-access article distributed under the terms of the Creative Commons Attribution Non-Commercial License (http://creativecommons.org/ licenses/by-nc/4.0) which permits unrestricted noncommercial use, distribution, and reproduction in any medium, provided the original work is properly cited. Copyright (c) 2017 by Korean Academy of Rehabilitation Medicine 


\section{INTRODUCTION}

Chronic kidney disease (CKD) is associated with various clinical problems such as uremia, malnutrition, anemia, hypertension, dyspnea, muscle weakness, dysesthesia, and even emotional stress including depression and anxiety. It involves not only the renal system but also the cardiovascular, respiratory, musculoskeletal, and nervous systems [1-4]. Recently, a growing body of evidence has suggested that physical performance limitations characterize many patients with CKD and affect the quality of their lives. Moreover, physical performance is associated with their outcomes, such as an increased risks for disability, hospitalization, and mortality [5-8]. Evaluation of physical performance is an important component of clinical care, and may provide useful information regarding health status in CKD patients.

Usual gait speed is one of the most valuable measurements of physical performance in the elderly and in patients with many chronic diseases. It is simple, quick, reproducible, inexpensive, and feasible for clinical use [9]. In the previous studies, diabetes is associated with decreased lower extremity function, such as a decrement in gait speed. Many factors, particularly including peripheral neuropathy (PN), may affect gait speed in diabetic patients [10-13]. CKD patients may also suffer the complications of both diabetic PN and uremic PN. Mitz et al. [14] reported that the severity of PN caused by diabetes is worse than that caused by primary renal disease such as nephritis. Ku et al. [2] reported the prevalence of PN in CKD patients with and without diabetes as being $61 \%$ and $27 \%$, respectively. Although the clinical criteria for distinction of both forms of PN is not obvious, it is reasonable to expect that there will be some differences in gait speed between CKD patients with and without diabetes. In addition, many factors-including fatigue, malnutrition, muscle wasting, musculoskeletal disorders and cardiovascular complications-have been proposed as potential contributors for gait speed decrement among CKD patients $[4,7,15]$. However, there were insufficient reports exploring the relationship between physical performance and PN in those patients. The aims of this study were (1) to compare overall physical function, including gait speed along with sensory, activity of daily living (ADL), and peripheral nerve function, between diabetic and nondiabetic CKD patients and (2) to investigate the associations between PN and other physical impairment of function in CKD patients. We hypothesized that (1) diabetic CKD patients might have a slower gait speed than would nondiabetic CKD patients and (2) among CKD patients, the severity of PN would be correlated with the degree of other physical impairment.

\section{MATERIALS AND METHODS}

\section{Subjects}

We screened a total of 276 adult CKD patients (over the age of 18) who received maintenance hemodialysis (HD) under the care of the renal unit at Samsung Changwon Hospital, Sungkyunkwan University School of Medicine from June to September 2015. We selected HD patients who met the following criteria: those who were undergoing regular maintenance HD (3 times/week) for at least 6 months; those with the ability to perform indoor walking independently; and those with the ability to understand and perform the tests of this study with informed consent. Patients were excluded if they had a history of neurologic disorder (e.g., stroke, Guillain-Barre syndrome, peripheral nerve injury), cardiovascular disorder (e.g., unstable angina, congestive heart failure), pulmonary disorder (e.g., chronic obstructive pulmonary disease, asthma), or musculoskeletal condition (e.g., recent fracture, amputation) that could influence walking ability or peripheral nerve function. Patients were also excluded if they had evidence of an active malignancy, if they had a history of falling down during the past 12 months, and if they or did not complete the tests of this study.

Sixty out of 276 patients satisfied our criteria and were enrolled in this study. They were divided into two groups, according to whether or not they had diabetes (Fig. 1). This study was approved by the Institutional Review Board of Samsung Changwon Hospital, Sungkyunkwan University School of Medicine, and all subjects gave informed consent.

\section{Methods \\ Usual gait speed}

Usual gait speed of the patients was measured over a $6-\mathrm{m}$ walkway. The lines of $0 \mathrm{~m}$ and $6 \mathrm{~m}$ were clearly marked by tape on a long, flat floor. The patients walked between the two lines at their usual pace, and the physician measured the time from each patient's first foot 


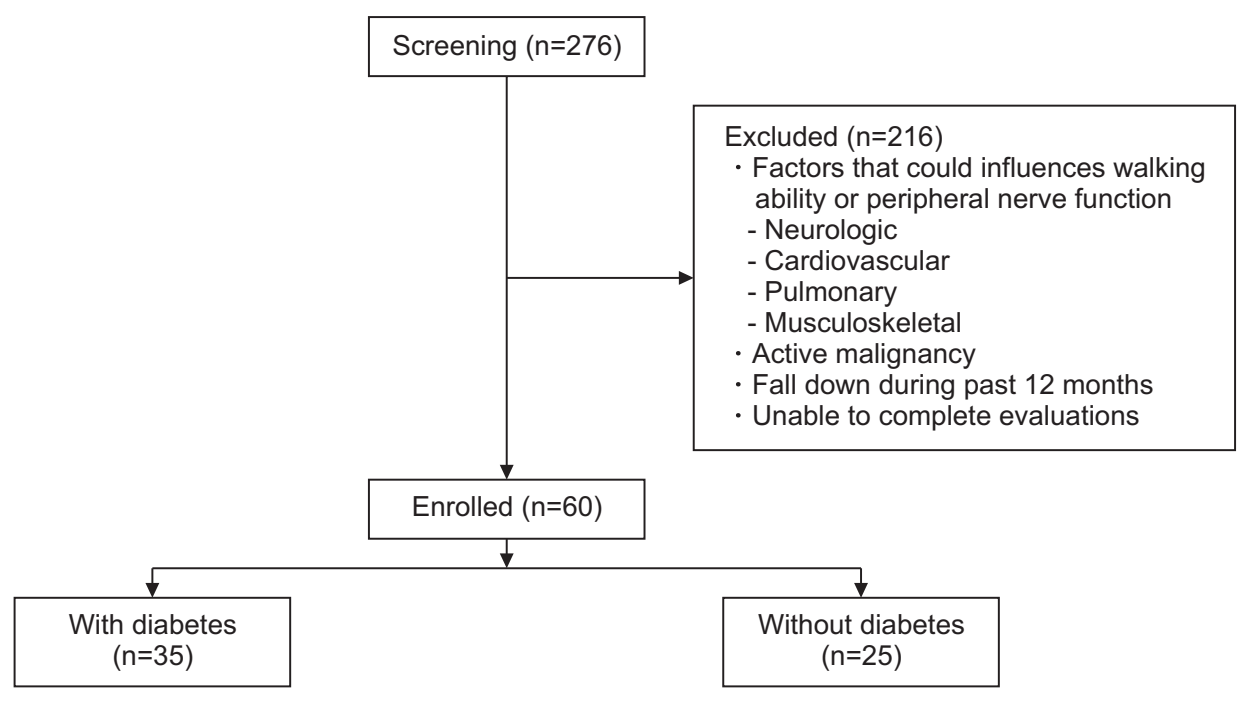

Fig. 1. Flowchart of subjects' inclusion. movements to the complete end-line cross of their last foot. After familiarization, the patients executed the walking test twice, and gait speed was calculated by determining the average time it took for them to walk $6 \mathrm{~m}$.

\section{Vibration perception threshold}

The vibration perception threshold (VPT) was assessed on both the index finger (VPT-F) and the great toe (VPTT) with TrueStar MMD-1000A (Medical Supply Co. Ltd., Wonju, Korea). Upper extremity without arteriovenous fistula for HD and lower extremity on the opposite side were selected for the evaluation. Stimulation intensity was adjusted automatically from 0 to $25 \mathrm{~Hz} / \mathrm{mV}$ and the threshold at which the patients perceived the stimulus was measured. After two trials, the average value was calculated. If the patients could not perceive the stimulus, the value was not recorded, and they were categorized as having 'failed' the test.

\section{Activity of daily living}

ADL difficulty was marked by patients' reports. If they needed assistance or were unable to do one or more of 4 tasks such as bathing, dressing, getting in and out of a chair, and walking around their home, they were considered as having ADL difficulty and were classified as being a member of that group [16].

\section{Electrodiagnostic evaluation}

Electrodiagnostic evaluation was performed using the Viking electromyography (EMG) system (Nicolet, Middleton, WI, USA). The function of median, peroneal, tibial, and sural nerves was assessed by means of the usual sensory and motor nerve conduction studies (NCS), F-wave study, and H-reflex study. Sensory NCS testing was performed with antidromic conduction. Needle EMG testing was performed on the tibialis anterior and the gastrocnemius muscles. An upper extremity without arteriovenous fistula for HD and a lower extremity on the opposite side were selected for the evaluation. H-reflex study and needle EMG test were performed bilaterally.

For statistical analysis, following the modified coding system of the Diabetes Control and Complications Trial (DCCT) [17], the severity of PN was coded as follows: 0, absent; 1 , mild; 2 , moderate; and 3, severe (Table 1).

\section{Data collection}

All the patients attended a questionnaire interview and performed a usual gait speed measurement test, a VPT test, and an electrodiagnostic evaluation after their first HD session of the week. In the VPT test, failed data were excluded in the between-group comparison, and the failed VPT was compared as another variable. In the electrodiagnostic evaluation, two variables (the presence of PN and its severity) were compared between the diabetic and nondiabetic groups.

ADL difficulty, demographic data (comprising age, sex, period of education, duration of HD, and body mass index [BMI]), past medical history (presence of hypertension and diabetes), and current medical condition (levels of creatinine clearance $\left[\mathrm{C}_{\mathrm{Cr}}\right]$, hemoglobin $[\mathrm{Hb}]$, albumin [Alb], and uric acid [UA]) were collected by questionnaire and by review of medical records. BMI and the results of 
Table 1. Criteria for abnormal electrodiagnostic evaluation and severity of peripheral neuropathy

\begin{tabular}{|ll}
\hline \multicolumn{1}{c}{ Criteria } & \multicolumn{1}{c}{ Description } \\
\hline Sural SNAP amplitude & $<5 \mu \mathrm{V}$ \\
Median SNAP amplitude & $<10 \mu \mathrm{V}$ \\
Peroneal CMAP amplitude & $<1 \mathrm{mV}$ \\
\hline Peroneal motor CV & $<40 \mathrm{~m} / \mathrm{s}$ \\
\hline Peroneal F-wave latency & Absent or $>55 \mathrm{~ms}$ \\
H-reflex & Absent bilaterally \\
\hline Needle EMG & Abnormal spontaneous activity in lower extremity muscles \\
Severity (code) & \\
\hline Absent (0) & \\
\hline Mild (1) & Criteria of sural SNAP amplitude plus at least two more criteria \\
\hline Moderate (2) & Mild plus two more criteria \\
Severe (3) & Moderate plus more criteria \\
\hline
\end{tabular}

SNAP, sensory nerve action potential; CMAP, compound muscle action potential; CV, conduction velocity; EMG, electromyography.

the blood test closest to the date of the evaluation were obtained from the records of the dialysis unit.

\section{Statistical analysis}

In this study, the first analysis was a comparison of the collected data between two groups. For the second analysis, correlations between the severity of PN and other variables (usual gait speed, VPT-F, VPT-T, and ADL difficulty) were identified.

After the screening for normality, subsequent data regarding age, period of education, duration of HD, BMI, levels of $\mathrm{CCr}, \mathrm{Hb}$, Alb and UA, usual gait speed, VPT-F, and VPT-T were reported in terms of mean \pm standard deviation and between-group comparisons of each of these variables was carried out with an independent t-test. The severity of PN was reported using median and interquartile range (IQR) data, which was compared using a Mann Whitney U-test. Sex, presence of HTN and PN, failed VPTF and VPT-T, and ADL difficulty were reported in terms of frequency and proportion and were compared by Pearson chi-square or Fisher exact tests. After between-group comparison, regression analysis for adjusting possible confounding factors was performed.

Associations between the severity of PN and other variables were identified using Spearman correlation analysis (for continuous variables, such as usual gait speed, VPTF, and VPT-T) and linear-by-linear association test (for categorical variables, such as ADL difficulty).

SPSS program ver. 18.0 for Windows (SPSS Inc., Chi- cago, IL, USA) was used for statistical analysis and any pvalues less than 0.05 were considered to be significant. Correlation coefficient (rho) was achieved in the Spearman correlation analysis.

\section{RESULTS}

A total of 60 patients, 35 with and 25 without diabetes (mean age $63.89 \pm 9.57$ vs. $56.4 \pm 14.67$ years, $\mathrm{p}=0.031 ; 24$ vs. 10 males, $\mathrm{p}=0.036)$, participated in this study. Except for age and sex, other demographic data and medical conditions did not show significant differences between the two groups. Usual gait speed (the primary outcome of this study) was significantly slower in the diabetic group than in the nondiabetic group $(0.87 \pm 0.21$ vs. $1.0 \pm 0.23 \mathrm{~m} /$ $\mathrm{s}, \mathrm{p}=0.029$ ). In the VPT test, the diabetic group showed a greater degree impairment of sensory function as compared to the nondiabetic group. VPT-F $(12.93 \pm 4.68$ vs. $9.17 \pm 4.81 \mathrm{~Hz}, \mathrm{p}=0.011)$, VPT-T $(15.33 \pm 6.24$ vs. $10.33 \pm 4.19$ $\mathrm{Hz}, \mathrm{p}=0.023)$ and the proportion of failed VPT-T ( $49 \%$ vs. $28 \%, p=0.036$ ) were significantly higher in the diabetic group than in the nondiabetic group. Only the proportion of failed VPT-F was not significantly differed between the two groups. The proportion of ADL difficulty was higher in the diabetic group, but not to a degree of statistical significance. In the electrodiagnostic evaluation, the diabetic group showed comparatively more impaired peripheral nerve function. They had more frequent $(77 \% \mathrm{vs}$. $28 \%, \mathrm{p}<0.001$ ) and more severe (median [IQR] severity, 
Table 2. Comparison of demographic data, medical condition, and the results of functional and electrodiagnostic evaluations between the diabetic and the nondiabetic groups

\begin{tabular}{|c|c|c|c|}
\hline & With diabetes $(n=35)$ & Without diabetes $(n=25)$ & p-value \\
\hline \multicolumn{4}{|l|}{ Demographics } \\
\hline Age (yr) & $63.89 \pm 9.57$ & $56.4 \pm 14.67$ & $0.031^{\mathrm{a})}$ \\
\hline Male & $24(69)$ & $10(40)$ & $0.036^{\mathrm{b})}$ \\
\hline Period of education (yr) & $10.14 \pm 3.20$ & $11.00 \pm 3.42$ & 0.324 \\
\hline Duration of HD (mo) & $54.26 \pm 49.61$ & $81.64 \pm 79.12$ & 0.105 \\
\hline Body mass index $\left(\mathrm{kg} / \mathrm{m}^{2}\right)$ & $23.57 \pm 3.64$ & $23.18 \pm 4.36$ & 0.704 \\
\hline \multicolumn{4}{|l|}{ Medical conditions } \\
\hline Hypertension & $21(60)$ & $19(76)$ & 0.195 \\
\hline Creatinine clearance $(\mathrm{mL} / \mathrm{min})$ & $9.41 \pm 2.80$ & $9.60 \pm 3.14$ & 0.811 \\
\hline Hemoglobin $(g / d L)$ & $10.47 \pm 1.12$ & $10.53 \pm 1.04$ & 0.843 \\
\hline $\operatorname{Albumin}(\mathrm{g} / \mathrm{dL})$ & $3.83 \pm 0.53$ & $3.82 \pm 0.47$ & 0.973 \\
\hline Uric acid (mg/dL) & $6.88 \pm 2.0$ & $7.52 \pm 1.82$ & 0.207 \\
\hline \multicolumn{4}{|l|}{ Functional evaluations } \\
\hline Usual gait speed (m/s) & $0.87 \pm 0.21$ & $1.00 \pm 0.23$ & $0.029^{a)}$ \\
\hline VPT-F (Hz) & $12.93 \pm 4.68$ & $9.17 \pm 4.81$ & $0.011^{\mathrm{a})}$ \\
\hline Failed VPT-F & $9(26)$ & $5(20)$ & 0.606 \\
\hline VPT-T (Hz) & $15.33 \pm 6.24$ & $10.33 \pm 4.19$ & $0.023^{\mathrm{a})}$ \\
\hline Failed VPT-T & $24(69)$ & $10(40)$ & $0.036^{\mathrm{b})}$ \\
\hline ADL difficulty & $17(49)$ & $7(28)$ & 0.109 \\
\hline \multicolumn{4}{|l|}{ Electrodiagnostic evaluation } \\
\hline PN & $27(77)$ & $7(28)$ & $0.000^{\mathrm{b})}$ \\
\hline Severity of PN & $2(1-3)$ & $0(0-1)$ & $0.000^{\mathrm{c})}$ \\
\hline
\end{tabular}

Values are presented as mean \pm standard deviation or number $(\%)$ or median (interquartile range).

HD, hemodialysis; VPT-F, vibration perception threshold for the index finger; VPT-T, vibration perception threshold for the great toe; $\mathrm{ADL}$, activities of daily living; $\mathrm{PN}$, peripheral neuropathy.

$\mathrm{p}<0.05$ by ${ }^{\mathrm{a})}$ independent $\mathrm{t}$-test, ${ }^{\mathrm{b})}$ Pearson chi-square test or Fisher exact test, ${ }^{\mathrm{c})}$ Mann-Whitney U-test.

$2[1-3]$ vs. $0[0-1], \mathrm{p}<0.001)$ PN than did the nondiabetic group (Table 2).

Because age and sex showed significant differences in between-group comparisons, the effects of both these factors and of diabetes on significant outcomes were coevaluated by means of multivariate regression analysis. Diabetes was found to be related with between-group differences in usual gait speed ( $\mathrm{p}=0.021)$, VPT-F $(\mathrm{p}=0.048)$, VPT-T ( $\mathrm{p}=0.045)$ and PN ( $\mathrm{p}=0.004)$, but age was also related with differences in usual gait speed $(\mathrm{p}<0.001)$ and VPT-T ( $p=0.010)$ (Table 3).

Spearman correlation analysis between the severity of PN and usual gait speed showed a negative linear correlation (rho $=-0.325, \mathrm{p}=0.011)$. By contrast, VPT-F (rho=0.346, p=0.018) and VPT-T (rho=0.4, p=0.035) were positively correlated with the severity of PN (Table 4).
Finally, in the linear-by-linear association test, patients with the more severe PN had a higher proportion of ADL difficulty $(\mathrm{p}=0.031)$ (Table 5).

\section{DISCUSSION}

In this cross-sectional single-cohort study, 60 adult CKD patients were enrolled and divided into two groups, according to whether or not they had diabetes. The diabetic group showed a slower gait speed, higher thresholds for vibration perception in both upper and lower extremities, and more frequent and severe $\mathrm{PN}$, as compared to the nondiabetic group. In addition, having a comparatively more severe $\mathrm{PN}$ was associated with having more impaired vibration perception and more frequent $\mathrm{ADL}$ difficulty, as well as a slower gait speed. In previous stud- 
Table 3. Multiple linear regression and logistic regression analysis (adjusting for age and sex)

\begin{tabular}{lll}
\hline $\begin{array}{c}\text { Dependent } \\
\text { variable }\end{array}$ & $\begin{array}{c}\text { Independent } \\
\text { variable }\end{array}$ & p-value \\
\hline Usual gait speed & Diabetes & $0.041^{\text {a) }}$ \\
& Age & $0.000^{\text {a) }}$ \\
\hline VPT-F & Sex & 0.464 \\
& Diabetes & $0.048^{\text {a) }}$ \\
\hline VPT-T & Age & 0.097 \\
& Sex & 0.337 \\
\hline & Diabetes & $0.045^{\text {a) }}$ \\
\hline PN & Age & $0.010^{\text {a) }}$ \\
\hline & Sex & 0.441 \\
& Diabetes & $0.004^{\text {b) }}$ \\
\hline
\end{tabular}

VPT-F, vibration perception threshold for the index finger; VPT-T, vibration perception threshold for the great toe; PN, peripheral neuropathy.

$\mathrm{p}<0.05$ by ${ }^{\text {a) }}$ multiple linear regression analysis, ${ }^{\text {b) }}$ logistic regression analysis.

ies, the patients' diabetes was proposed as being a potential contributor to decreased lower extremity and peripheral nerve function $[10,18,19]$. Of note, the present study showed that similar impacts of diabetes also appeared in the physical function of patients with CKD, including in gait speed, sensory perception, ADL difficulty, and peripheral nerve function.

In the present study, diabetic CKD patients showed a significantly slower gait speed than did those without diabetes (Table 2). Importantly, our patients were composed homogenously in terms of renal replacement therapy and ambulatory function. We included only those patients with a history of having undergone regular maintenance HD for at least 6 months and we excluded those with variable disorders or conditions which could influence their walking ability. In addition, a direct comparison of gait speed between diabetic and nondiabetic CKD patients might more clearly identify the impact of diabetes on their physical performance. Bohannon et al. [18] also suggested the adverse effects of diabetes as being contributing factors to the worsening of a walking impairment in kidney transplant candidates. However, they enrolled subjects with both HD and peritoneal dialysis, as well as those with previous transplants-and even those
Table 4. Associations between the severity of peripheral neuropathy and the findings of other functional evaluations

\begin{tabular}{lcc}
\hline & $\begin{array}{c}\text { Correlation } \\
\text { coefficient (rho) }\end{array}$ & p-value \\
\hline Usual gait speed & -0.325 & $0.011^{\text {a) }}$ \\
VPT-F & 0.346 & $0.018^{\text {a) }}$ \\
VPT-T & 0.400 & $0.043^{\text {a) }}$ \\
\hline
\end{tabular}

VPT-F, vibration perception threshold for the index finger; VPT-T, vibration perception threshold for the great toe. ${ }^{a)} \mathrm{p}<0.05$ by Spearman correlation analysis.

Table 5. Association between the severity of PN and the presence of difficulty in performing ADL

\begin{tabular}{ccccc}
\hline \multirow{2}{*}{$\begin{array}{c}\text { ADL } \\
\text { difficulty }\end{array}$} & \multicolumn{4}{c}{ Severity of PN } \\
\cline { 2 - 5 } & Absent & Mild & Moderate & Severe \\
\hline Absent & 20 & 2 & 7 & 7 \\
Present & 6 & 2 & 8 & 8 \\
\hline
\end{tabular}

Values are presented as the number of patients.

$\mathrm{PN}$, peripheral neuropathy; ADL, activities of daily living. $\mathrm{p}=0.031$ by linear-by-linear association test.

with amputation, fracture, and joint replacement in their lower extremities. In a large prospective cohort study of data from the US Renal Data System, CKD patients classified in the fastest walk speed category (36.3\%) were least likely to have diabetes; while those in the slowest category (65\%) and the category of being 'unable to walk' (77\%) had a profoundly higher proportion of diabetes [7]. They measured gait speed as a predictor of patients' outcomes, but they did not compare it or evaluate the risk factor for its decrement.

Many factors, including fatigue, malnutrition, muscle wasting, musculoskeletal disorders, and cardiovascular complications, have been proposed as being potential contributors for gait speed decrements among CKD patients $[4,7,15]$. In the present study, PN was suggested as being another one such contributor. The severity of PN, which had been clearly diagnosed and graded by electrodiagnostic evaluation, was found to negatively correlate with gait speed (Table 4). The diabetic PN is one of the most frequent and the most severe of complications of diabetes, as is uremic PN for CKD. In particular, PN has been identified as being the main individual contributing factor for lower extremity disability in patients with dia- 
betes and among the elderly [10-13]. We concluded that the results of our correlation analysis showed the likelihood of PN as being one of the contributing factors for the physical disability of CKD patients.

On the other hand, there were significant differences in gait speed, sensory function, and peripheral nerve function between diabetic and nondiabetic CKD patients. Because our patients maintained regular HD for more than 6 months, the interrupted progression of uremic PN could be one possible explanation for these differences. Ogura et al. [20] reported that the most HD patients showed no remarkable electrophysiological changes in uremic neuropathy during HD. In addition, Thomas [21] reported that the number of patients exhibiting clinical features of uremic neuropathy has recently decreased because of the technical improvements in HD. Additional research, with risk evaluations, is necessary to clearly compare the impacts of diabetic and uremic PN with physical disability.

Presently, our two groups showed demographic differences, such as in terms of age and sex (Table 1). Although diabetes was still related with a decrement in gait speed after adjusting for age and sex, age was also related with a slower gait speed and a higher VPT-T (Table 3). Similarly, in a previous US Renal Data System study, the cumulative prevalence of a comparatively slow gait speed among HD patients was found to increase with age [7]. Furthermore, in another comparison study between type 2 diabetes patients and nondiabetic controls, the former group showed a shorter duration of dialysis regardless of careful age matching [22]. These findings presumably mean that age is another major factor for gait speed decrement among CKD patients, and that diabetic CKD patients begin HD at an older age with more impaired physical performance. For this reason, introducing rehabilitation programs for these patients is a recommendation which should be actively implemented.

There were a number of potential limitations in our study. A major limitation was that the study design involved comparisons between CKD patients with or without diabetes. Although this study clearly showed the impact of diabetes on physical disability in CKD patients, the relative risk of diabetes itself could not be analyzed. In addition, demographic characteristics were not controlled and they differed between the two groups. Finally, impaired sensory function-as measured by VPT test- ing-might be a cause of gait dysfunction. Further studies, including controlled group design or a large sample with regression analysis for individual risk evaluation, will be needed.

In CKD patients with regular HD, gait speed along with sensory and peripheral nerve functions all were significantly impaired whether or not they have diabetes, and the severity of PN was negatively correlated with their gait speed, sensory function, and ADL function. Adverse effects of diabetes also negatively impacted the physical performance of CKD patients. In addition, the physical disability of those patients might be attributable to PN and its severity. Therefore, electrodiagnostic evaluation as well as physical performance measurements should be performed on patients with CKD, and an appropriate rehabilitation program should be provided, according to their results. Further studies are needed to evaluate the beneficial effects of exercise programs on physical performance in this patient population in order to improve patient care.

\section{CONFLICT OF INTEREST}

No potential conflict of interest relevant to this article was reported.

\section{REFERENCES}

1. Kasper DL, Fauci AS, Hauser SL, Longo DL, Jameson JL, Loscalzo J. Harrison's principles of internal medicine. 19th ed. New York: McGraw-Hill Companies; 2016. p. 1811-21.

2. Ku do Y, Park YS, Chang HJ, Kim SR, Ryu JW, Kim WJ. Depression and life quality in chronic renal failure patients with polyneuropathy on hemodialysis. Ann Rehabil Med 2012;36:702-7.

3. Palamidas AF, Gennimata SA, Karakontaki F, Kaltsakas G, Papantoniou I, Koutsoukou A, et al. Impact of hemodialysis on dyspnea and lung function in end stage kidney disease patients. Biomed Res Int 2014;2014:212751.

4. Johansen KL, Shubert T, Doyle J, Soher B, Sakkas GK, Kent-Braun JA. Muscle atrophy in patients receiving hemodialysis: effects on muscle strength, muscle quality, and physical function. Kidney Int 2003;63:2917. 
5. Painter P, Marcus RL. Assessing physical function and physical activity in patients with CKD. Clin J Am Soc Nephrol 2013;8:861-72.

6. Roshanravan B, Robinson-Cohen C, Patel KV, Ayers E, Littman AJ, de Boer IH, et al. Association between physical performance and all-cause mortality in CKD. J Am Soc Nephrol 2013;24:822-30.

7. Kutner NG, Zhang R, Huang Y, Painter P. Gait speed and mortality, hospitalization, and functional status change among hemodialysis patients: a US renal data system special study. Am J Kidney Dis 2015;66:297304.

8. Reese PP, Cappola AR, Shults J, Townsend RR, Gadegbeku CA, Anderson C, et al. Physical performance and frailty in chronic kidney disease. Am J Nephrol 2013;38:307-15.

9. Cummings SR, Studenski S, Ferrucci L. A diagnosis of dismobility: giving mobility clinical visibility: a Mobility Working Group recommendation. JAMA 2014;311:2061-2.

10. Volpato S, Blaum C, Resnick H, Ferrucci L, Fried LP, Guralnik JM, et al. Comorbidities and impairments explaining the association between diabetes and lower extremity disability: the Women's Health and Aging Study. Diabetes Care 2002;25:678-83.

11. Resnick HE, Stansberry KB, Harris TB, Tirivedi M, Smith K, Morgan P, et al. Diabetes, peripheral neuropathy, and old age disability. Muscle Nerve 2002;25:4350 .

12. Resnick HE, Vinik AI, Schwartz AV, Leveille SG, Brancati FL, Balfour J, et al. Independent effects of peripheral nerve dysfunction on lower-extremity physical function in old age: the Women's Health and Aging Study. Diabetes Care 2000;23:1642-7.

13. Inzitari M, Carlo A, Baldereschi M, Pracucci G, Maggi S, Gandolfo C, et al. Risk and predictors of motorperformance decline in a normally functioning population-based sample of elderly subjects: the Ital- ian Longitudinal Study on Aging. J Am Geriatr Soc 2006;54:318-24.

14. Mitz M, Di Benedetto M, Klingbeil GE, Melvin JL, Piering W. Neuropathy in end-stage renal disease secondary to primary renal disease and diabetes. Arch Phys Med Rehabil 1984;65:235-8.

15. Johansen KL, Doyle J, Sakkas GK, Kent-Braun JA. Neural and metabolic mechanisms of excessive muscle fatigue in maintenance hemodialysis patients. Am J Physiol Regul Integr Comp Physiol 2005;289:R805-13.

16. Gill TM, Hardy SE, Williams CS. Underestimation of disability in community-living older persons. J Am Geriatr Soc 2002;50:1492-7.

17. The Diabetes Control and Complications Trial Research Group. The effect of intensive diabetes therapy on the development and progression of neuropathy. Ann Intern Med 1995;122:561-8.

18. Bohannon RW, Smith J, Hull D, Palmeri D, Barnhard R. Deficits in lower extremity muscle and gait performance among renal transplant candidates. Arch Phys Med Rehabil 1995;76:547-51.

19. Chiles NS, Phillips CL, Volpato S, Bandinelli S, Ferrucci L, Guralnik JM, et al. Diabetes, peripheral neuropathy, and lower-extremity function. J Diabetes Complications 2014;28:91-5.

20. Ogura T, Makinodan A, Kubo T, Hayashida T, Hirasawa Y. Electrophysiological course of uraemic neuropathy in haemodialysis patients. Postgrad Med J 2001;77:451-4.

21. Thomas PK. Screening for peripheral neuropathy in patients treated by chronic hemodialysis. Muscle Nerve 1978;1:396-9.

22. Boucek P, Saudek F, Pokorna E, Vitko S, Adamec M, Koznarova R, et al. Kidney transplantation in type 2 diabetic patients: a comparison with matched nondiabetic subjects. Nephrol Dial Transplant 2002;17: 1678-83. 